\title{
Study on Dynamic Prediction of Thermal Response for Aircraft Device Cabin
}

\author{
Shichao Liu ${ }^{1}$, Liping Pang ${ }^{1}$ \\ ${ }^{1}$ School of Aeronautic Science and Engineering, BeiHang University, XueYuan Road \\ No.37,HaiDian District, Beijing, China \\ Iscbuaa@163.com
}

Keywords: Dynamic simulation; Thermal network model; Thermal environment; Aircraft cabin

\begin{abstract}
The effect of aerodynamic heating is obvious for hypersonic aircrafts. Its temperature change of cabin electronic devices is obvious under this severe working condition. Therefore, for this type of aircraft, it is important to design the thermal protecting system and evaluate the impact on electrical devices. It is valuable for the designer to obtain the results of the dynamic prediction of thermal response accurately in advance. The aim of this paper is to establish a thermal network model based on Kirchhoff's circuit laws through an analogy of electrical and thermal systems. The simulation model is solved by using numeric simulation method. The presented thermal network model can realize the quick thermal response of the wall insulation and cabin devices due to aerodynamic heating, and then obtain the transient thermal simulation results of aircraft internal device. Comparisons with the reliable commercial Sinda/Fluent model show that the presented model has a good prediction accuracy. Therefore, the presented model can realize the accurate prediction of the transient heat transfer inside the cabin in a short time.
\end{abstract}

\section{Introduction}

Serious problems of aerodynamic heating will happen when aircrafts flight at the speed of hypersonic. Aerodynamic heating is one of the key technology problems in the development of thermal protection and control for high-speed aircraft. The accuracy predicting of thermal environment for aircraft cabin has significant meaning for the development of hypersonic aircraft. Whether the heat characteristics of electronic devices can be accurate predictions are the premise of designing thermal protection systems. Therefore, the study of method predicting thermal environment for hypersonic aircraft is very important for the design of aircrafts.

The CFD numerical method widely used is mature, but with the increasing scale and complex structure of research model, the quality of the mesh generation has great impact for results and computation speeds when the model in the course of numerical calculation 错误!未找到引用源。. It will cost time to regain the calculation results if the data parameters need to be modified in engineering application.

In this paper, thermal network models derive from an analogy of electrical and thermal systems. According to the actual physical model of aircraft, it can be divided into several units. Each unit should have uniform temperature, heat flux and the effective radiation, each component of aircraft regards as the hot node. Radiation, conduction and convection heat transfer between units can be summed to heat transfer process connected by a variety of thermal resistance between nodes. A thermal network is formed if this kind of relationship between nodes using graphics to express. It has the relation of analogy between thermal network and electrical network in the diagram and mathematical model. For example: the thermal flux corresponding to the electric current, the thermal resistance corresponding to the resistance, and the thermal capacitance corresponding to the capacitance. Therefore, the thermodynamic equation of each node can be established by the means of using Kirchhoff's circuit laws 错误!未找到引用源。.

Compared with the CFD numerical methods, the requirement of thermal network method for mesh-accuracy is reduced greatly. The mesh can be divided into very thick so that the thermal behavior is represented with smaller equation systems. Therefore, the method of this paper 
proposed greatly improves the calculation speed compared with the CFD numerical methods.

The aim of this paper is to show the thermal response of cabin electronic devices and wall based on the theory of resistance and capacitance by the means of node thermal network analysis method, compare its simulation result to the reliable commercial Sinda/Fluent mode, and evaluate its suitability for an aircraft.

\section{Layout for aircraft internal load}

The study object of this paper is an aircraft cabin. In order to achieve the requirements of computational efficiency, the cabin model is simplified to represent thermal behavior with smaller equation systems and less input parameters. The physical model is showed in Fig.1. The model represents all important relevant thermal devices of aircraft cabin.

During modeling, all surfaces are involved in the heat transfer of heat conduction, convection and radiation, and angles are not considered in the model. Each electronic device is simplified to a node. The heat sources are effective in the entire. It is assumed that the air temperature in the thermal zone is homogeneous, and the air node is defined to one node which has convective heat transfer with equipment, floor and cabin wall.

As shown in Fig. 1, thermal nodes include each device nodes, air node, inner wall node, outer wall node, floor node, and insulation layer node.



Fig.1. Simplified cabin model

\section{Node thermal network}

The nodes of protective layers. The body of the hypersonic aircraft applied the protective structure. The most outside layer is the high temperature resistant metals. The inner layer is a thermal protective material. The most inner layer is the body structure of aircraft.

Temperature of insulation layer. The thermal resistance of the insulation material is big, so the internal temperature gradient is accordingly large. In this paper, a heat conduction differential equation is used. In general, the radius of the aircraft cabin wall is relatively large, and its thickness is far smaller than radius. Thus, the heat conduction of insulation layer can be simplified as one-dimensional heat conduction through plane wall. It is assumed that the physical properties of solids do not vary with temperature change. The differential equations of its heat conduction is:

$$
\frac{\partial T_{s, i}(\tau)}{\partial \tau}=a_{w} \frac{\partial^{2} T_{s, i}(\tau)}{\partial x^{2}}, 0 \leq x \leq \delta, 0 \leq \tau \leq \tau_{0} .
$$

where $T_{s, i}, \tau_{0}, \delta$ are the temperature of insulation layer, times, thickness of insulating layer, computing time, respectively; $s$ represents in the insulation layer; $i$ is the thermal node number, $i=1$, $2, \ldots ., I ; x$ is the coordinate along the wall thickness direction; $\alpha_{w}$ is thermal diffusivity; $\lambda, \rho, c_{\mathrm{p}}$ are the thermal conductivity, density, specific heat capacity, respectively.

For the heat conduct differential equations in heat insulation layer, the heat transfer model can not be directly transformed into a thermal network model, Eq. 1 is discrete by using finite difference method, and Then Eq. 2 can be obtained:

$$
\frac{\partial T_{s, i}^{l}(\tau)}{\partial \tau}=a_{w} \frac{T_{s, i}^{l-1}(\tau)-2 T_{s, i}^{l}(\tau)+T_{s, i}^{l+1}(\tau)}{\Delta x^{2}} .
$$

where $l$ is represented in the number of divided layers of insulation, $l=1,2, . ., L$. 
The concept of generalized heat capacity is established:

$$
C_{s}=m c_{p}=\rho v c_{p} .
$$

where $m$ is the mass of insulation node; $v$ is the volume of insulation node, respectively. In addition, the following simplified equation is defined:

$$
\frac{\lambda \cdot v_{s}}{\Delta x^{2}}=\frac{\lambda \cdot A_{i}}{\Delta x}=D_{s} .
$$

where $A_{i}$ is the contacting area between nodes. Eq. 2 can be translated into an equivalent thermal equation:

$$
C_{\mathrm{s}} \frac{\partial T_{s, i}^{l}(\tau)}{\partial \tau}=D_{s}\left[T_{s, i}^{l+1}(\tau)+T_{s, i}^{l-1}(\tau)-2 T_{s, i}(\tau)\right]
$$

Temperature of inner wall. The temperature of inner wall is high due to aerodynamic heating, thus, it cannot neglect the influence of heat radiation. Because the temperature of the inner wall is approximate uniform, thus, the average radiation temperature of the inner wall is introduced in this paper.

$$
\bar{T}_{w}=\left(\frac{1}{\sum_{i=1, \ldots, I} A_{i}} \cdot \sum_{i=1, \ldots, I} A_{i} \cdot T_{w, i}^{4}\right)^{1 / 4} .
$$

Where $w$ is inner wall.

The irregular radiation from the inner wall to devices, which is transformed into a uniform radiation by using the average radiation temperature. It is necessity to linearize the radiative part when build the thermal network model, Because the radiative part is nonlinear which cannot transformed into the form of Resistance and capacitance.

A common simplification of this equation defines a heat transfer coefficient for the radiate part 错 误!未找到引用源。

It is assumed that there are two objects, the radiate heat transfer can be expressed with Stefan's law:

$$
q=\varepsilon \sigma\left(T_{1}^{4}-T_{2}^{4}\right)
$$

where $\varepsilon$ is emissivity and it depends on the temperature and surface condition of object; $\sigma$ is Stefan's constant. If the temperature difference between object 1 and 2 is small enough, and their average temperature is $T_{m}$, the radiate part can be simplification in Eq. 9:

$$
\mathrm{q}=\varepsilon \sigma\left(T_{1}^{4}-T_{2}^{4}\right)=\varepsilon \sigma\left(T_{1}^{3}-T_{1}^{2} T+T_{1} T_{2}+T_{2}^{3}\right)\left(T_{1}-T_{2}\right) \approx 4 \varepsilon \sigma T_{m}^{3}\left(T_{1}-T_{2}\right) .
$$

By defining a constant mean surface temperature, the radiate heat transfer coefficient can be assumed as constant:

$$
\begin{aligned}
& Q=A h_{r}\left(T_{1}-T_{2}\right) . \\
& h_{r}=4 \varepsilon \sigma T_{m}^{3} .
\end{aligned}
$$

The thermal equation of the inner wall is

$$
\begin{aligned}
& \frac{C_{i}}{2} \frac{d T_{w, i}}{d \tau}=\sum_{i=1,2 . I} D_{s, w}\left(T_{s, i}^{L}-T_{w, i}\right)-\sum_{i=1,2 . I} H_{w, a i r}\left(T_{w, i}-T_{a i r}\right)-4 \sum_{j=1,, J} \gamma_{i} B T_{m}^{3}\left(\bar{T}_{w}-T_{E, j}\right)-\sum_{i=1,2 . I} D_{w, f}\left(T_{w, i}-T_{f}\right) . \\
& C_{i}=m_{i} \cdot c_{p, i},
\end{aligned}
$$




$$
\begin{aligned}
& D_{s, w}=A \cdot h, \\
& H_{w, \text { air }}=A \cdot h, \\
& B=\varepsilon \cdot \sigma \cdot A .
\end{aligned}
$$

where $C_{i}$ is generalized capacity of node; $D_{s, w}$ is generalized heat transfer coefficient between insulation layer and inner wall; $H_{w, \text { air }}$ is generalized convection heat transfer coefficient between inner wall and air; $h$ is contact heat transfer coefficient; $A$ is the heat transfer surface; $B$ is generalized radiation heat transfer coefficient; $\varepsilon_{1}$ and $\varepsilon_{2}$ are the constants of $0.05 ; A_{E, j}$ and $A_{w}$ are the area of electronic devices and inner wall respectively; $T_{s, i}, T_{w, i}, T_{a i r}, T_{f}$ and $T_{E, j}$ are the temperatures of insulation layer, inner wall, air, floor, and electronic devices respectively.

The nodes of cabin load. The thermal equation of the device nodes is

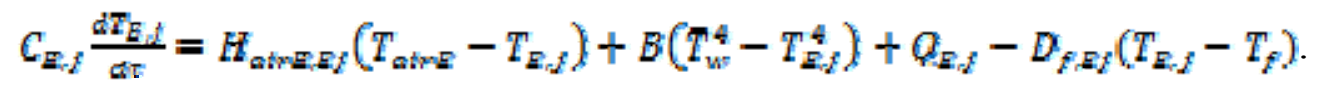

The thermal equation of the of floor nodes is

$$
C_{f} \frac{d T_{f}}{d \tau}=H_{\text {airE }, f}\left(T_{a i r E}-T_{f}\right)+D_{w 1, f}\left(T_{w, 1}-T_{f}\right)+D_{w i, f}\left(T_{w, i}-T_{f}\right)+\sum_{j=1, . ., J} D_{E j, f}\left(T_{E, j}-T_{f}\right) .
$$

\section{Aerodynamic heating}

The X-34 technology demonstrator is typical aircrafts based on the rocket propulsion system in aircraft technical verification project. Surface temperature is the known input parameters. A typical change as shown in Fig.2, the flight time is $600 \mathrm{~s}$ 错误!找到引用源。

\section{Thermal network model}

The key points of the thermal network method are that the nodes can be correctly selected and the heat transfer process can be complete analysis. The selected nodes on the one hand can correctly reflect the essence of physical model; on the other hand, it not only can make the model get reasonable simplification, but also can save the calculation cost effectively. This requires a detailed analysis of the heat transfer mode between the various devices of the cabin before selected nodes ${ }^{[5]}$.

In general, the number and location of nodes should be chosen according to the requirement of accuracy and the rationality of the computation time. The selection of nodes must be as far as possible to make the units represented by the nodes are isothermal. This is more important for nodes which must consider thermal property change with temperature. The heat flow between adjacent nodes should be uniform distribution as far as possible ${ }^{[2]}$.

Through the above analysis, the heat transfer network model is established by the analogy circuit model. The thermal network model is showed in fig. 3


Fig.2. Simplified cabin model

Fig. 3. Thermal network picture 


\section{Numerical results and discussion}

The thermal network model was implemented in Matlab, its result was compared with the results of large commercial software Sinda which has been widely used. The following several nodes were be selected for comparing with each other: $T_{s 1}, T_{s 2}, T_{f}, T_{E 1}, T_{E 2}, T_{E 3,}$ and $T_{\text {air }}$.

In order to compare the predicted values of the two models, a common analysis based on the coefficient of determination $\left(R^{2}\right)$ was be used. For values of $R^{2}$ near 1 , the estimate provides a good approximation to the date set. In addition to the coefficient of determination, Root-mean-square error is used to measure absolute deviations. For values of RMSE near 0, it indicates that the prediction accuracy of the model is higher.

$$
\begin{gathered}
R^{2}=1-\frac{\sum_{i=1}^{n}\left(y_{i}-\hat{y}_{i}\right)^{2}}{\sum_{i=1}^{n}\left(y_{i}-\bar{y}_{i}\right)^{2}}, \\
R M S E=\left[\frac{\sum_{i=1}^{n}\left(y_{i}-\hat{y}_{i}\right)^{2}}{N}\right]^{0.5} .
\end{gathered}
$$

where $y_{i}$ is date of sinda model; $\widehat{y_{i}}$ is date of thermal network model $; \bar{y}_{i}$ is mean value of sinda model; $N$ is The number of date

Table 1 shows the comparison results of insulation layer, inner wall, air, floor, devices, respectively. Because of the large temperature gradient of the insulation layer, the maximum RMSE is $7.688^{\circ} \mathrm{C}$, which can be reduced by increasing the number of nodes for heat insulating layer. For simulation results of inner wall, air and floor, the maximum RMSE is maintained at around 2, the coefficient of determination is more than 0.98 . For the temperature prediction of various electronic devices, the coefficients of determination is all above 0.99 .

Table 1. Results of error analyses

\begin{tabular}{|c|c|c|c|c|c|}
\hline cabin & $\operatorname{RMSE}\left({ }^{\circ} \mathrm{C}\right)$ & $\mathrm{R}^{2}$ & cabin & $\operatorname{RMSE}\left({ }^{\circ} \mathrm{C}\right)$ & $\mathrm{R}^{2}$ \\
\hline Insulation layer 1 & 2.964 & 0.9827 & air & 1.033 & 0.9822 \\
\hline Insulation layer 2 & 7.688 & 0.9998 & device 1 & 0.077 & 0.9955 \\
\hline Inner cabin wall & 2.300 & 0.9713 & device 2 & 0.090 & 0.9993 \\
\hline Floor & 1.215 & 0.9969 & device 3 & 0.125 & 0.9992 \\
\hline
\end{tabular}

Comparisons of the simulation results of new model with Sinda model are shown in Fig.4, Fig. 5, from which it can be seen that the results predicted by the two models have smaller deviation. The temperature curve predicted by the new model agree with the Sinda model very well.

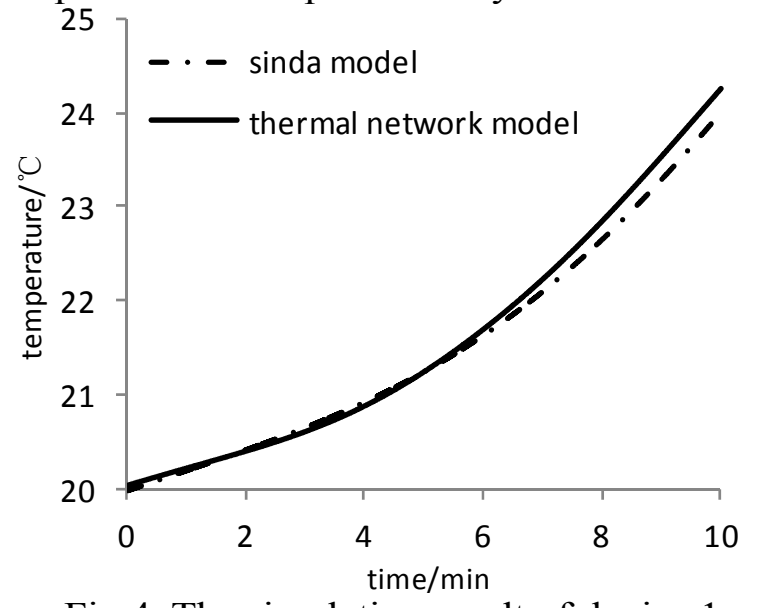

Fig.4. The simulation result of device 1



Fig.5.The simulation result of device 2 


\section{Summary}

The thermal network model proposed in this paper can represent a thermal environment by thermal resistances and capacitances with low computation costs. The applicability of the new model in thermal environment is validated by comparing with Sinda model. The results show that the new model can be used to predict the dynamic thermal response of aircraft cabin. Because the two models have different processing methods for the radiation, thus, the simulation results of the two models have some deviations. In addition to the thermal insulation layer has a big temperature gradient, which lead to the larger prediction error, the rest of the simulation results have good simulation accuracy. The thermal network model proposed in this paper can achieve the simulation requirements of the thermal environment of the aircraft cabin.

\section{Acknowledgment}

The research is sponsored by the Aviation Science Foundation of China (20130251005).

\section{Reference}

[1] Y. Liu, L.L. Jiang: submitted to Journal of Aerospace Science and Technology (2010).

[2] Z.H. Li: Satellite Thermal Control Technology(China Aerospace Publications, Beijing 2007).

[3] X.T. Cheng, X.G. Liang: submitted to Journal of Heat and Mass Transfer (2011).

[4] Z.Q. Hou, J.G. Hu: submitted to Jou rnal of Thermal Control Technology in Spacecraft (2007).

[5] M. Ishizuka, S. Nakagawa, T. Hachiga: Mathematical Sciences. Forum Vol. 28(2009), p. 667

[6] S.P.Hou, Z. Tao and S.J. Han: submitted to Journal of Aerospace Power(2010). 